\title{
Suppressing of Power Line Artifact From Electroencephalogram Measurements Using Sparsity in Frequency Domain
}

OPEN ACCESS

Edited by:

Yizhang Jiang,

Jiangnan University, China

Reviewed by:

Zhe Yang,

Dongguan University of Technology,

China

Xin Zhang,

Southwest Jiaotong University, China

Yu Wang,

Xi'an Jiaotong University, China

*Correspondence:

Min Zhang

minzhang@xidian.edu.cn

$\mathrm{NaXu}$

xuna1313@163.com

Specialty section:

This article was submitted to

Brain Imaging Methods,

a section of the journal

Frontiers in Neuroscience

Received: 21 September 2021

Accepted: 08 October 2021

Published: 28 October 2021

Citation:

Tan J-L, Liang Z-F, Zhang R, Dong $Y-Q$, Li G-H, Zhang $M$, Wang $H$

and Xu N (2021) Suppressing

of Power Line Artifact From

Electroencephalogram Measurements

Using Sparsity in Frequency Domain.

Front. Neurosci. 15:780373.

doi: 10.3389/fnins.2021.780373

\author{
Jin-Lin Tan 1,2, Zhi-Feng Liang', Rui Zhang ${ }^{2}$, You-Qiang Dong ${ }^{1}$, Guang-Hui Li', \\ Min Zhang ${ }^{1 *}$, Hai Wang ${ }^{1}$ and $\mathrm{Na} \mathrm{Xu}^{3 *}$
}

${ }^{1}$ School of Aerospace Science and Technology, Xidian University, Xi'an, China, ${ }^{2}$ Shaanxi Aerospace Technology Application Research Institute Co., Ltd, Xi'an, China, ${ }^{3}$ The Second Affiliated Hospital of Xiamen Medical College, Xiamen, China

Electroencephalogram (EEG) plays an important role in brain disease diagnosis and research of brain-computer interface $(\mathrm{BCl})$. However, the measurements of $E E G$ are often exposed to strong interference of power line artifact (PLA). Digital notch filters (DNFs) can be applied to remove the PLA effectively, but it also results in severe signal distortions in the time domain. To address this problem, spectrum correction (SC) based methods can be utilized. These methods estimate harmonic parameters of the PLA such that compensation signals are produced to remove the noise. In order to ensure high accuracy during harmonic parameter estimations, a novel approach is proposed in this paper. This novel approach is based on the combination of sparse representation (SR) and SC. It can deeply mine the information of PLA in the frequency domain. Firstly, a ratio-based spectrum correction (RBSC) using rectangular window is employed to make rough estimation of the harmonic parameters of PLA. Secondly, the two spectral line closest to the estimated frequency are calculated. Thirdly, the two spectral lines with high amplitudes can be utilized as input of RBSC to make finer estimations of the harmonic parameters. Finally, a compensation signal, based on the extracted harmonic parameters, is generated to suppress PLA. Numerical simulations and actual EEG signals with PLA were used to evaluate the effectiveness of the improved approach. It is verified that this approach can effectively suppress the PLA without distorting the time-domain waveform of the EEG signal.

Keywords: EEG, brain imaging, sparse representation, noise reduction, salient feature enhancement

\section{INTRODUCTION}

In the measurement of Electroencephalogram (EEG), differences in voltages from distinct sites of the brain are recorded over a period of time (Hernández-Ronquillo et al., 2020; Sun et al., 2020). The EEG signal contains abundant information related to physiological, psychological and pathological activities of the brain. Therefore, the analysis of EEG signals is of vital importance in the clinical diagnosis and treatment of many diseases, such as Alzheimer's disease (Faiman et al., 2021), depression (Miao et al., 2021), idiopathic epilepsy and psychogenic non-epileptic seizures (Sharma G. et al., 2021). On the other hand, EEG also plays an important role in the research of brain-computer interface (BCI). BCI is referred to as a non-invasive way of human-machine 
interface between the brain and exterior devices (Dagdevir and Tokmakci, 2021). BCI does not rely on conventional neurotransmission pathways (Deshpande et al., 2017). Nowadays, many brain imaging methodologies have been adopted by the researchers, such as EEG, magnetic resonance imaging (MRI) and functional MRI (fMRI; Hong et al., 2018). Among the above mediums, EEG is regarded as the most convenient one due to its mobility and lower cost.

In medical applications, owing to non-stationary characteristics of EEG features and differences among individual measurements, the analysis of EEG signals is comparatively complicated and difficult (An et al., 2021). The traditional signal analysis method based on fast Fourier transform (FFT) cannot meet the requirements of EEG signal analysis. In order to reduce excessive professional requirements for medical staff to identify EEG, deep learning (DL) based methods, such as convolutional neural network (CNN; Cao et al., 2019; Prathaban and Balasubramanian, 2021), are introduced to ensure intelligent understanding of EEG signals, especially for large scale datasets. Aiming at achieving robust recognition ability, researchers have designed novel DL neural networks with distinguished types of network architecture, input formulation, and activation function (Saegh et al., 2021). Li proposed an FFT-based deep feature learning method for EEG classification ( $\mathrm{Li}$ and Chen, 2021). Shankar proposed a DL based epileptic seizure detection algorithm by using 2D recurrence plot images generated from EEG signals for specific brain rhythms (Shankar et al., 2021). Jana studied an efficient seizure prediction technique based on the combination of $\mathrm{CNN}$ and a novel technique of channel reduction (Jana and Mukherjee, 2021). According to the above studies, researchers also attached significant attentions in deriving special features from raw EEG signals because it can help improve the accuracy and efficiency of the DL neural networks.

In spite of the versatility of EEG signal in clinical applications and brain imaging science, it is reported that the salient features within the EEG signals are weak in energy. On the other hand, a variety of artifacts are likely to be incorporated during acquisitions of EEG signals. To investigate the essential physiological information corrupted by these noisy artifacts, pre-processing based on signal processing techniques is indispensable. Among the many sources of interference, the power line artifact (PLA), at the frequency of 50 or $60 \mathrm{~Hz}$, is almost inevitable in "field" or mobile EEG measurements outside the lab (Leske and Dalal, 2019). The presence of PLA can significantly affect the analysis and extraction of essential features from EEG recordings. Although shielding of the environment has been recognized as an effective way to reduce PLA in the lab, it is often impractical in scenarios of natural environments. Therefore, alternative solutions, hardware based or software based, must be adopted to address this PLA problem. In comparison, the advantages of software-based solutions are more prominent because they are more flexible and easier to be realized. In state-of-the-art researches, signal processing tools, such as digital notch filters (DNFs; Piskorowski, 2013), short time Fourier transform (STFT; Huang et al., 2019), wavelet transform (WT; He et al., 2015; Huang et al., 2021; Sharma S. et al., 2021), and empirical mode decomposition
(EMD; Taran et al., 2018), have been utilized to remove the PLA. However, according to theoretical investigations, the above techniques can be interpreted as digital filters with specific passing band in the frequency domain (Wu and Huang, 2004). Owing to the problems of energy leakage and picket-fence effect (PFE) in canonical Fourier transform, such kinds of digital filters will cause non-ignorable distortions in filtered results, whereas the same problems also occur in hardwarebased solutions. To overcome this side effect, a feasible way is to construct a compensation signal with high precision. According to engineering experiences, PLA can be modeled as a sinusoidal component consisting of a simple harmonic wave. While, a sinusoidal component can be uniquely determined by harmonic parameters of amplitude, frequency and phase. Therefore, to retrieve the harmonic parameters become the essential task in PLA removal.

Sparse representation (SR) is a comparatively new development of signal expansion ( $\mathrm{Li}$ and Chen, 2008). It expresses an input signal in terms of linear combinations of atoms from a dictionary. It has achieved tremendous successes in various engineering applications (Wang et al., 2016; Yang et al., 2020; Zhang et al., 2021). Conventional SRs are implemented using iterative algorithms and may require large computational resources (Cao et al., 2021; Chen et al., 2021; An et al., 2022). If the efficiency of SR algorithm can be improved, it will effectively expand the scope of its use in medical engineering (Yang et al., 2019; Collazos-Huertas et al., 2020; Cury et al., 2020). In this paper, to overcome the signal distortion effect in PLA removal of EEG signals, we propose a novel approach based on the combination of SR and spectrum correction (SC). SC methods can identify the harmonic parameters based on the information in the frequency domain. It will be shown in this paper that for a sinusoidal component, the SC can be regarded as a numerical algorithm to realize SR via a non-iterative way. In ratio-based spectrum correction (RBSC) algorithms, the performance of harmonic parameter extraction is determined by signal-to-noise ratios of baseline spectral lines (BSSLs). Taking this phenomenon into account, a dual-step correcting algorithm (DSCA) is put forward. Firstly, a RBSC, using the rectangular window, is employed for preliminary identification of harmonic parameters. Secondly, two BSSLs with high SNR are calculated. Thirdly, another RBSC is employed to estimate the harmonic parameters with higher precision. On the basis of the estimated harmonic parameters, a compensation signal is reconstructed. PLA removal by subtracting the compensation signal from the original EEG signal can avoid the side effect of signal distortion. Analysis using numerical simulation and measured signal shows that the proposed method is effective, efficient, and can suppress waveform distortion.

\section{MATERIALS AND METHODS}

\section{Mechanism of Signal Distortion in Electroencephalogram Pre-processing}

To ensure high performance of BCI, noises in EEG signals should be suppressed using pre-processing based on hardware-based 
or software-based solutions. However, severe signal distortions may be produced by such pre-processing. The theory of Fourier transform can be used to explain the reason of this side effect. An EEG signal $x(t)$, corrupted by the PLA, can be expressed as $x(t)=$ $e e g(t)+p l a(t)$, where eeg $(t)$ and pla $(t)$ stands for the salient EEG features and PLA, respectively. In most cases, the PLA component can be modeled as a sinusoidal component, expressed aspla $(t)=A_{\text {pla }} \cdot \cos \left(2 \pi f_{\text {pla }} t+\varphi_{\text {pla }}\right)$. These harmonic parameters $\left(A_{\text {pla }}, f_{\text {pla }}, \varphi_{\text {pla }}\right)$ are crucial to determine the waveform of PLA. In actual analysis of a dynamic process, the digitization of a physical quantity requires the signal to have a finite sampling frequency $\left(f_{s}\right)$ and a finite sampling number $(N)$ (Figure 1A). That is to say, after digitization, $x(t)$ should be expressed as $x[n]=x((n-$ $1) \cdot \Delta t$ ), where $n=0,1, \ldots, N-1$ and the spatial interval is denoted as $\Delta t=1 / f_{s}$. The FFT converts an input signal from the time domain into a counterpart in the frequency domain. In numerical implementations of FFT, a cluster of sinusoidal waves are used to decompose the input signals.

$$
X[\ell]=F F T\{x[n]\},
$$

where $X[\ell](\ell=0,1 \ldots, N-1)$ represents the complex-valued coefficient at the frequency of $(\ell-1) \cdot f_{s} / N$. The FFT spectrum is also a sampling of actual Fourier spectrum of $x(t)$. If the frequency $f_{\text {pla }}$ does not belong to the set $\left\{(\ell-1) \cdot f_{s} / N \mid \ell=\right.$ $0,1 \ldots, N-1\}$, the problem of energy leakage and PFE occur (Chen et al., 2019). In such circumstance, the FFT spectrum of $\{p l a[n]\}$ is dense in frequency domain (Figure 1B). The energy of the noisy component $\{$ pla $[n]\}$ leaks across the entire frequency domain. A simple harmonic wave in time domain becomes a broad band signal in the frequency domain. Therefore, the harmonic parameters cannot be estimated using any single spectral line in the FFT spectrum (Figure 1C).

Wavelet transform and EMD are important developments of the classical FFT. However, they are essentially digital filters with specific passing band in the frequency domain (Figure 1D). By using them, only a part of the PLA spectrum can be suppressed. The residual contents after digital filtering still leads to side effect of signal distortion. This phenomenon is similar to Gibbs phenomenon in Fourier analysis. Therefore, it can also be summarized as a pseudo-Gibbs phenomenon (PGF) in multiscale analysis.

\section{Fundamentals of Sparse Representation}

Sparse representation has become a hot research topic in the field of signal processing during the past two decades. SR aims at expressing an input signal $x$ as a linear combination of atoms from an over-complete dictionary $\Phi$.

$$
x=\Phi c=\left[\begin{array}{llll}
\varphi_{1} & \varphi_{2} & \cdots & \varphi_{M}
\end{array}\right]\left(\begin{array}{c}
c_{1} \\
c_{2} \\
\vdots \\
c_{M}
\end{array}\right),
$$

where $\varphi_{i} \in \Phi(i=1,2, \ldots, M)$ and $M$ is the number of atoms in $\Phi$. For EEG signal analysis, the variables $x$ and $\varphi_{i}$ are supposed to be column vectors of dimension $K$. The dimension of $\Phi$ in matrix form is $K \times M$. Different from a basis, the condition $M \times K$ should be satisfied for an SR dictionary $\Phi$. To satisfy the demand of sparsity, most coefficients in the set $\left\{c_{i}\right\}$ are approximate or equal to zero.

To obtain a sparse solution $\hat{c}$, we need to solve the following optimization problem.

$$
\hat{c}=\operatorname{argmin}\|c\|_{0}, \quad \text { s.t. } x=\Phi c
$$

where the L0-norm $\|c\|_{0}$ calculates the number of non-zero terms in $\hat{c}$. However, this problem has proven to be NP-hard. To deal with this problem, the L1-norm $\|c\|_{1}=\sum_{i=1}^{K}\left|c_{i}\right|$ can be introduced to derive a feasible solution. Therefore, the problem in Eq. (3) is formulated as below.

$$
\hat{c}=\operatorname{argmin}\|c\|_{1}, \text { s.t. } x=\Phi c
$$

In practical applications, owing to the existence of measurement noises, the constraint condition $x=\Phi c$ is usually too strict that may results in no solution. A relaxed L2-norm constraint, allowing a maximum error of $\varepsilon$, is more convenient.

$$
\hat{c}=\operatorname{argmin}\|c\|_{1} \text {, s.t. }\|x-\Phi c\|_{2} \leq \varepsilon
$$

To solve the above optimization problem, iterative algorithms, such as matching pursuit and basis pursuit, can be utilized. From the above argument, the solution of $\hat{c}$ depends on the choosing of a proper dictionary $\Phi$. Therefore, a critical problem of SR in engineering applications lies in the selection a proper over-complete dictionary.

\section{Spectrum Correction for Sinusoidal Component}

Fast Fourier transform is extensively applied to investigate the component of $x(t)=A_{c} \cdot \cos \left(2 \pi f_{c} t+\varphi_{c}\right)$, which appears as a simple harmonic wave in a dynamic process. According to the fundamentals of digital signal processing, if the condition $f_{s} / f_{c} \in$ $\mathrm{N}^{+}$is satisfied, the information of the PLA noise can be uniquely revealed by one spectral line, whose frequency is exactly $f_{c}$, in the FFT spectrum. However, this condition is difficult to meet in most cases because the value of $f_{c}$ can be a complicated decimal. In such circumstances, SCs, based on the information in FFT spectra, should be adopted. In this section, we take the rectangular window function as an example to illustrate the SC algorithm.

In FFT analysis, the spectral resolution is defined as $\Delta f=$ $1 /(N \cdot \Delta t)$. Spectral lines are uniformly spaced by one single spectral resolution in the frequency axis. According to the algorithm of RBSC, a pair of spectral lines whose frequencies differ by one spectral resolution are utilized to estimate the harmonic parameters. For ease of argument, we name the two spectral lines as BSSLs. The frequencies of BSSL are denoted by $f_{l}$ and $f_{r}$, and they are belonging to the spectral grid set $\{(\ell-1)$. $\left.f_{s} / N \mid \ell=0,1 \ldots, N-1\right\}$. To estimate harmonic parameters of a sinusoidal component at the frequency of $f_{c}$, the following relationship is satisfied.

$$
f_{c}-0.5 \leq f_{l}<f_{c}<f_{r} \leq f_{c}+0.5
$$

The concept of normalized index of spectral line is used to indicate the frequency of a spectral line in FFT spectrum. Let 
A Signal of finite sampling frequency and finite sampling length

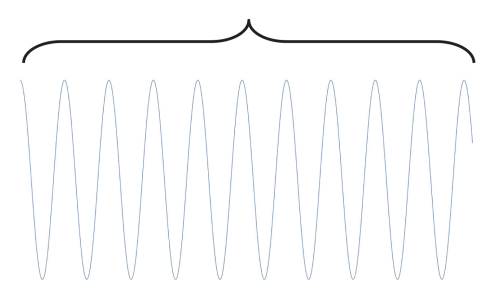

Fast Fourier Transform

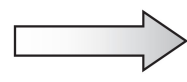

FFT spectrum

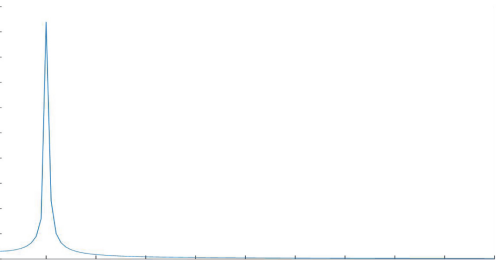

Frequency

C

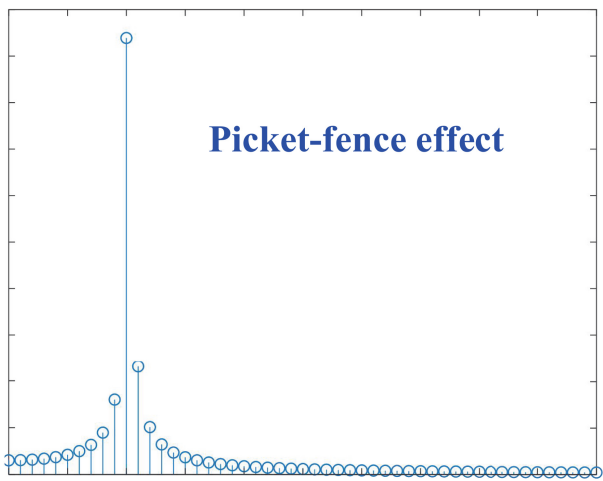

Frequency

D

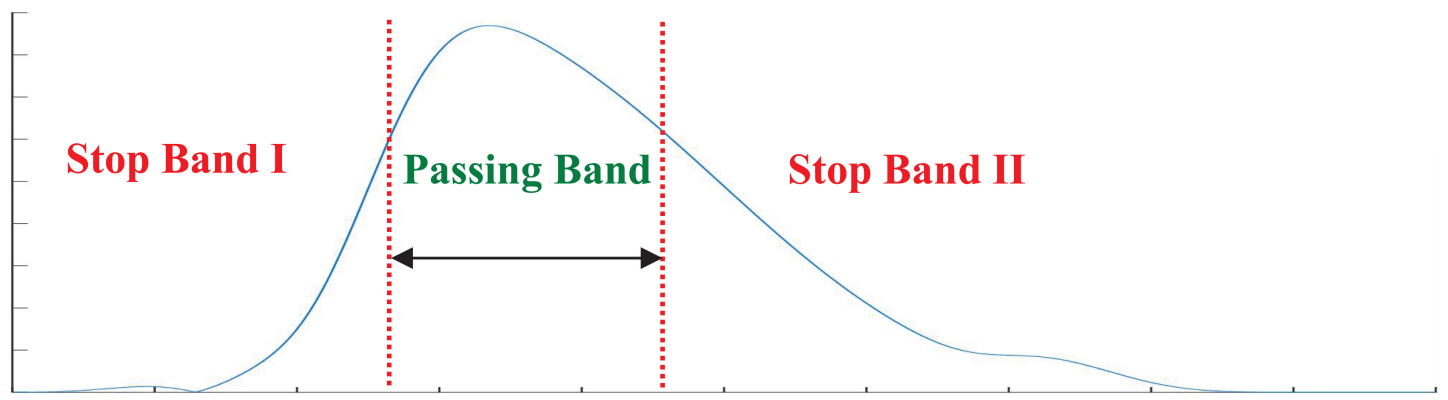

Frequency

FIGURE 1 | (A) Digitization of dynamic processes with finite sampling frequency and a finite sampling number; (B) FFT spectrum of a sinusoidal component; (C) the energy leakage problem; (D) the picket-fence effect (PFE) and the filtering characteristics of a digital filters from Daubechies wavelet basis (Mallat, 1989; Chen et al., 2012).

$Y_{k}$ be the complex number associated with the spectral line whose frequency is $(k-1) \cdot \Delta f, y_{k}$ and $\arg \left(Y_{k}\right)$ can be used to represent amplitude and argument of the $Y_{k}$. Figure 2A shows the amplitudes $\left(y_{l}\right.$ and $\left.y_{r}\right)$ of BSSLs in RBSC. The normalized frequency shift is calculated by $\Delta k=\left(f_{c}-f_{l}\right) / \Delta f$. In RBSC, the ratio between $y_{l}$ and $y_{r}$ can be used to calculate $\Delta k$ conveniently, which is implemented based on the information fusion of BSSLs. An indicator of characteristic ratio for the $\mathrm{SC}$ can be defined as $R=y_{-} l / y_{r}$. Using the characteristic ratio, the normalized error $\Delta k$ can be calculated as below.

$$
\Delta k=-\frac{1}{1+R}=-\frac{y_{k}}{y_{k}+y_{k+1}}
$$

Then the estimated harmonic parameters $\left(\tilde{A}_{c}, \tilde{f}_{c}\right.$, and $\left.\tilde{\varphi}_{c}\right)$ of the investigated sinusoidal component can be derived as below.

$$
\left\{\begin{array}{l}
\tilde{A}_{c}=\frac{\pi \cdot \Delta k \cdot y_{k}}{\sin (\pi \cdot \Delta k)} \\
\tilde{f}_{c}=(k+\Delta k) \cdot \Delta f \\
\tilde{\varphi}_{c}=\arg \left(Y_{k}\right)-\pi \cdot \Delta k
\end{array}\right.
$$

The sinusoidal component is supposed to be produced using the estimated harmonic information. In summary, the RBSC produces a harmonic atom that is very similar to the original PLA. This harmonic atom can be treated as a special dictionary with $M=1<<K$. This is the relationship between SC and SR for sinusoidal component analysis. 


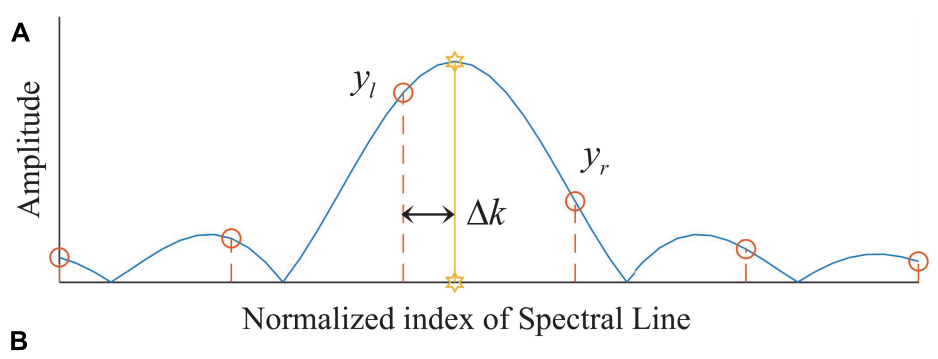

D

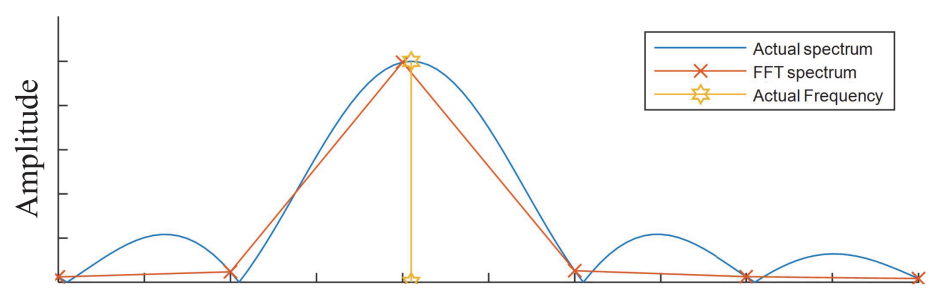

C

Normalized index of Spectral Line
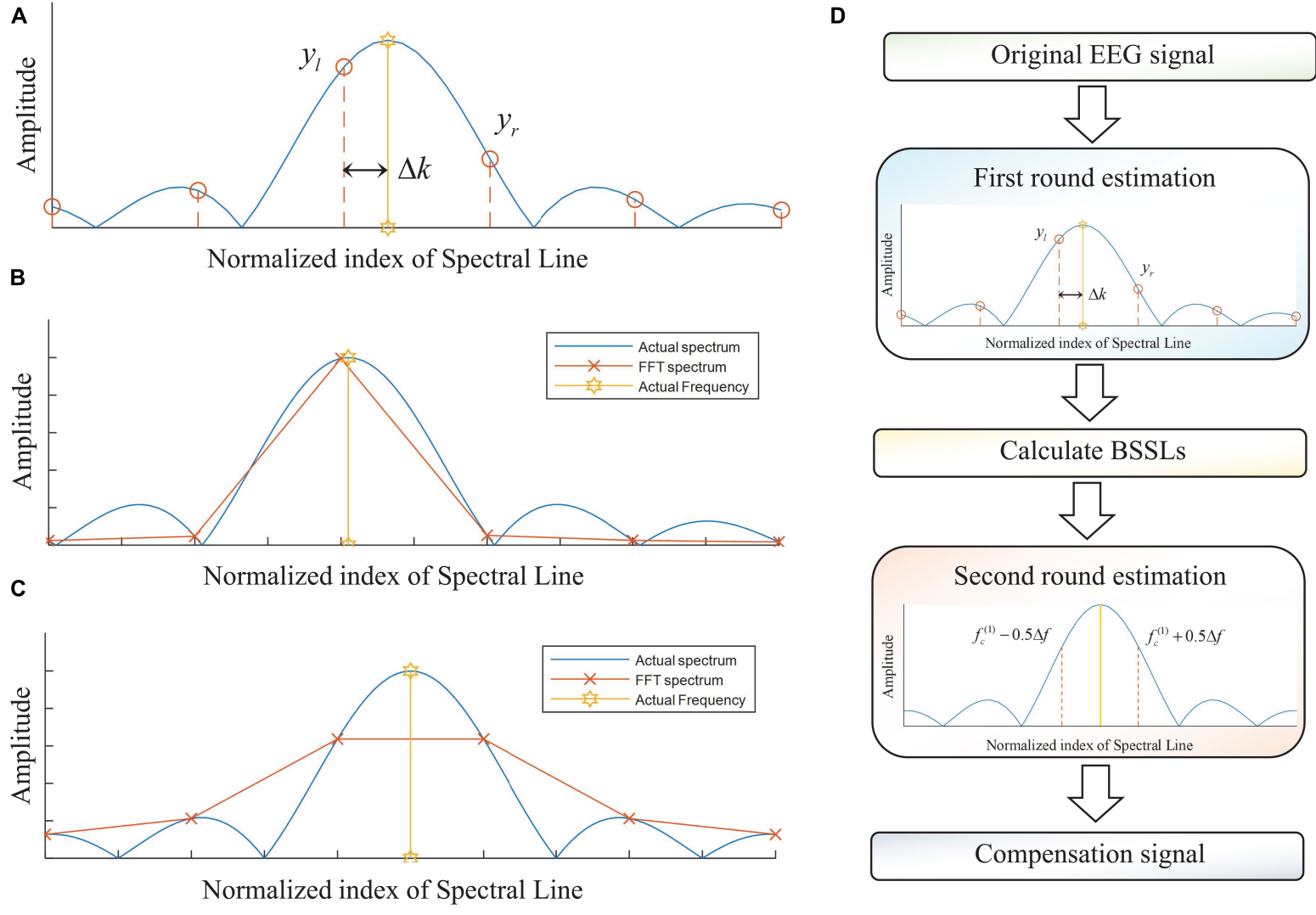

FIGURE 2 | (A) Mathematical model for the RBSC; (B) RBSC for small normalized frequency error; (C) RBS for normalized frequency error $\Delta k=1 / 2$; and (D) the flowchart of the DBSC.

\section{The Proposed Method}

The RBSC is an effective method to estimate harmonic parameters of a sinusoidal component. It utilizes information of BSSLs in FFT spectrum to estimate the harmonic parameters. In actual dynamic measurements, the information of BSSLs is also affected by noises. Therefore, high SNR are important to ensure estimations of high precision. As the value of $\Delta k$ approaches zero, the SNR of $y_{r}$ reduces significantly if measurement noise exists (Figure 2B). As a result, significant estimation errors can be produced for the harmonic parameters. As a compromise, we expect $\Delta k$ to be approximately $1 / 2$ such that both of $y_{l}$ and $y_{r}$ are of high amplitudes to resist the measurement noises (Figure 2C). To address this problem, a DSCA is proposed. The key idea of DSCA is the numerical implementation of spectral correction using BSSLs of high amplitudes. The flowchart of the DBSC is shown in Figure 2D.

Step 1. Apply FFT for the input signal $x(t)=A_{c} \cdot \cos \left(2 \pi f_{c} t+\right.$ $\left.\phi_{c}\right)$.

$$
x(t) \stackrel{F F T}{\longrightarrow} X(k)
$$

Step 2. First round estimation of harmonic parameter in $x(t)$ using RBSC.

$$
X(k) \stackrel{R B S C}{\longrightarrow} A_{c}^{(1)}, f_{c}^{(1)}, \varphi_{c}^{(1)}
$$

Step 3. Calculate BSSLs at frequencies of $f_{c}^{(1)}-0.5 \cdot \Delta f$ and $f_{c}^{(1)}+0.5 \cdot \Delta f$ based on $f_{c}^{(1)}$ and the FFT spectrum.

$$
\left\{\begin{array} { l } 
{ f _ { c } ^ { ( 1 ) } } \\
{ X ( k ) }
\end{array} \rightarrow \left\{\begin{array}{l}
f_{c}^{(1)}-0.5 \cdot \Delta f \\
f_{c}^{(1)}+0.5 \cdot \Delta f
\end{array}\right.\right.
$$

Step 4. Second round estimation of the harmonic parameters $\left(\tilde{A}_{c}, \tilde{f}_{c}, \tilde{\varphi}_{c}\right)$ using RBSC.

$$
\left\{\begin{array}{l}
f_{c}^{(1)}-0.5 \cdot \Delta f \stackrel{R B S C}{\longrightarrow} A_{c}^{(2)}, f_{c}^{(2)}, \varphi_{c}^{(2)} \\
f_{c}^{(1)}+0.5 \cdot \Delta f
\end{array}\right.
$$

Step 5. The harmonic parameters are corrected as below.

$$
\left\{\begin{array}{l}
\tilde{A}_{c}=A_{c}^{(1)} \\
\tilde{f}_{c}=f_{c}^{(2)} \\
\tilde{\phi}_{c}=\varphi_{c}^{(2)}
\end{array}\right.
$$

Step 6. A compensation signal can be produced using the corrected harmonic information.

$$
\tilde{x}(t)=\tilde{A}_{c}^{(1)} \cdot \cos \left(2 \pi \tilde{f}_{c}^{(2)} t+\tilde{\varphi}_{c}^{(2)}\right)
$$

According to the above algorithm, no iteration is employed. The original sinusoidal component is retrieved with errors 

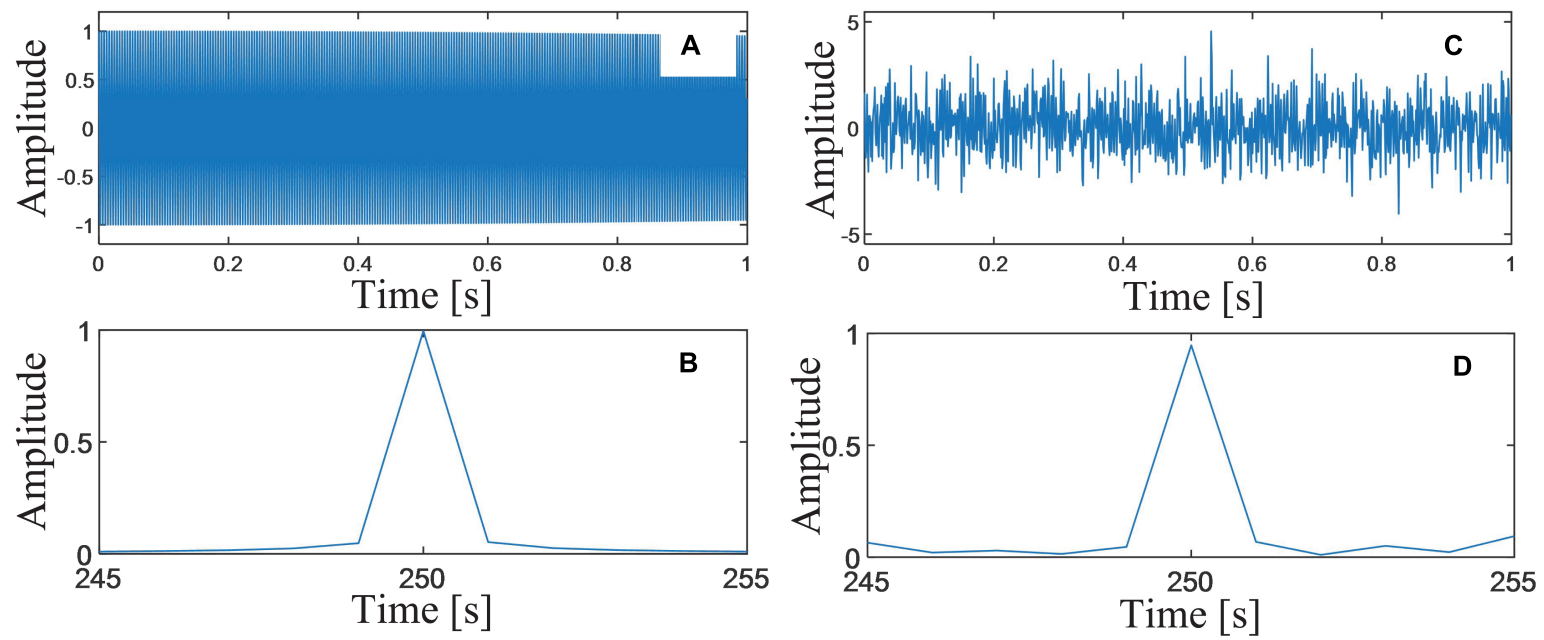

FIGURE 3 | (A) Waveform of the simulated PLA; (B) FFT spectrum of the simulated PLA; (C) the simulated PLA with noises (SNR = OdB); and (D) FFT spectrum of the simulated PLA with noises.

$\|x(t)-\tilde{x}(t)\|>0$. Only one atom, learned by the spectral corrections, is used to reconstruct $x(t)$. This can be seen as a special form of SR for sinusoidal component.

\section{RESULTS}

\section{Numerical Simulations}

To verify the enhancement of the proposed DSCA, numerical simulations are employed in this sub-section. A signal, consisting of PLA (pla $(t)$, Figure 3A) and noises $(n(t))$, is simulated. Without loss of generality, the harmonic parameters are set as $A_{\text {pla }}=1, f_{\text {pla }}=250.05 \mathrm{~Hz}$, and $\varphi_{\text {pla }}=0$. A white Gaussian noise $n(t)$ is added to the simulated signal, and the SNR is set as $0 \mathrm{~dB}$ (Figure 3C). Both of the sampling frequency and the sampling number are set as 1,000. The spectral resolution of the signal in spectral domain is calculated as $\Delta f=1$ (Figures 3B,D). In FFT spectrum of pla $(t)$, the frequencies of BSSLs are 250 and $251 \mathrm{~Hz}$. Their amplitudes are $y_{l}=$ 0.996 and $y_{r}=0.052$. The normalized frequency shift is $\Delta k=$ 0.05 , which is very closed to zero. The harmonic parameters are corrected as $A_{\text {pla }}=1, f_{\text {pla }}=250.05 \mathrm{~Hz}$, and $\phi_{\text {pla }}=0$. The estimation accuracy is perfect when there is no noise. While in the spectrum of $s(t), y_{l}=0.947$ and $y_{r}=0.068$. The harmonic parameters are corrected as $A_{\text {pla }}=0.9539, f_{\text {pla }}=250.067 \mathrm{~Hz}$, and $\phi_{\text {pla }}=-0.010$. It can be seen significant estimation errors occur due to noises.

For the proposed DSCA, the first-round estimation of the frequency is $f_{c}^{(1)}=250.067$. The frequencies of BSSLs are $f_{l}=249.567$ and $f_{r}=255.067$. The harmonic parameters are corrected as $A_{\text {pla }}=1.073, f_{\text {pla }}=250.040 \mathrm{~Hz}$, and $\varphi_{\text {pla }}=-0.006$. It can be seen that the estimation errors of the frequency and the phase are reduced significantly, while estimation error of the phase increases. The same rule of error has also been verified in other independent repeated experiments. That is the reason why we use the estimated harmonic parameters in different rounds of estimations.

$$
s(t)=p l a(t)+n(t)
$$

\section{Case Study of Noisy Electroencephalogram Signals}

In this sub-section, actual measurements of EEG signals are employed to further verify the performance of the proposed DSCA. The EEG datasets, we used in this paper, were made available to the public by Neurology \& Sleep Centre, Hauz Khas, New Delhi. The employed datasets contain EEG records from clinical studies concerning epilepsy. A record of EEG time series is selected from the datasets (Figure 4A). The sampling frequency is $200 \mathrm{~Hz}$, and the sampling number is 1,000 . In acquisition of the EEG time series, a band-pass digital filter with the passing band of $[0.5,70] \mathrm{Hz}$ was applied. To simulate the PLA, an additive noisy component is synthesized with the parameters $A_{\text {pla }}=50$, $f_{\text {pla }}=250.05 \mathrm{~Hz}$, and $\varphi_{\text {pla }}=0$ (Figure 4B). The FFT spectra of the two components are shown in Figure 4C. A noisy EEG signal is formed by directly superimposing the two components. The SNR of the investigated signal is $3.430 \mathrm{~dB}$.

The proposed DSCA is applied to remove the PLA. The harmonic parameters are corrected as $A_{\text {pla }}=50.006$, $f_{\text {pla }}=250.050 \mathrm{~Hz}$, and $\varphi_{\text {pla }}=-0.023$. Using these estimated parameters, the compensation signal can be reconstructed and subtracted from the synthesized signal. The denoised signal is plotted in Figure 4D. From the appearances of the two waveforms, they are very similar. The correlation coefficient of the two signals are computed as 1.00, indicating that the filtering result is satisfactory. The FFT spectra of the denoised signal and the original noisy EEG signal are shown in Figure 4E. The difference only exists in a very narrow frequency band around $50 \mathrm{~Hz}$.

As comparison, a DNF based method is also used to process the noisy signal (Chen et al., 2012). The denoised signal is shown 

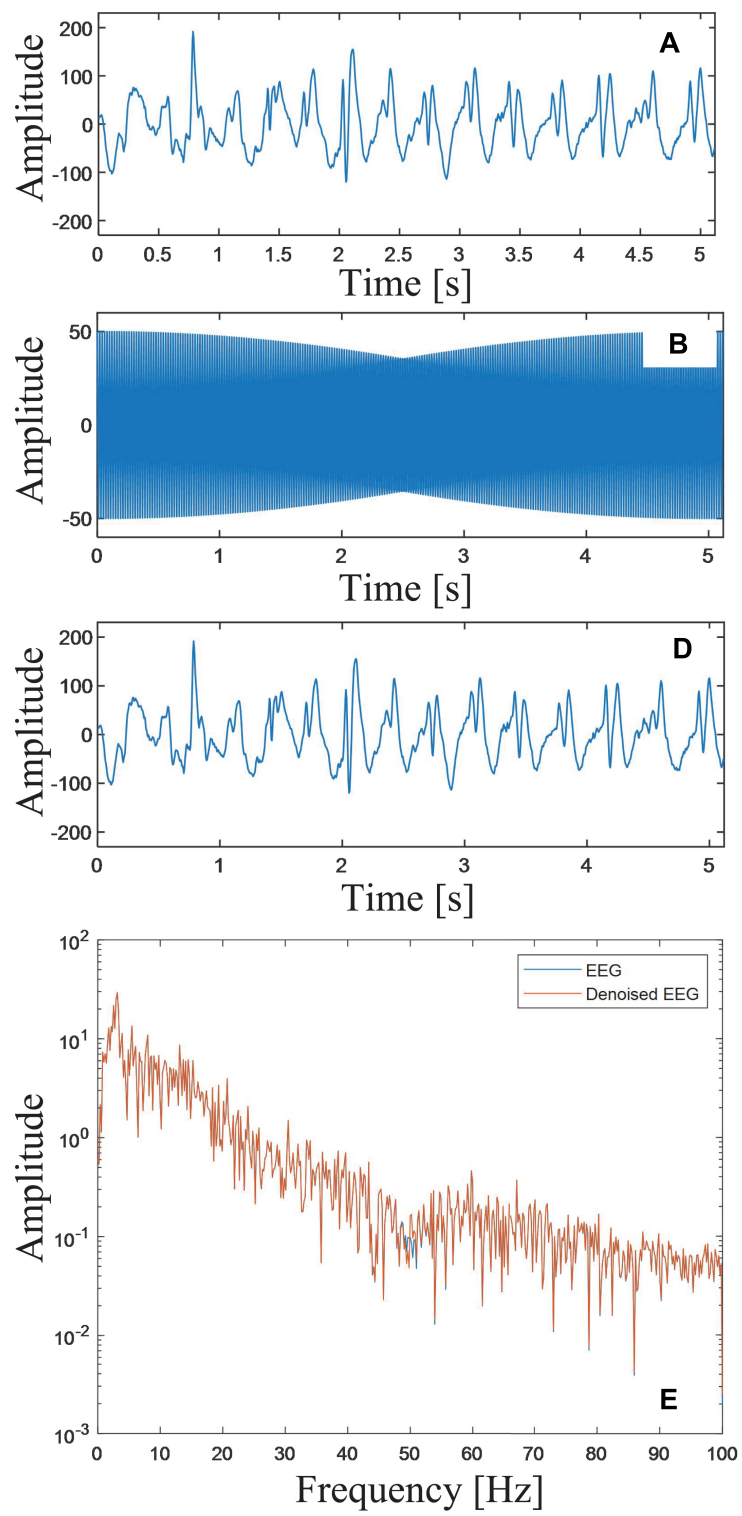
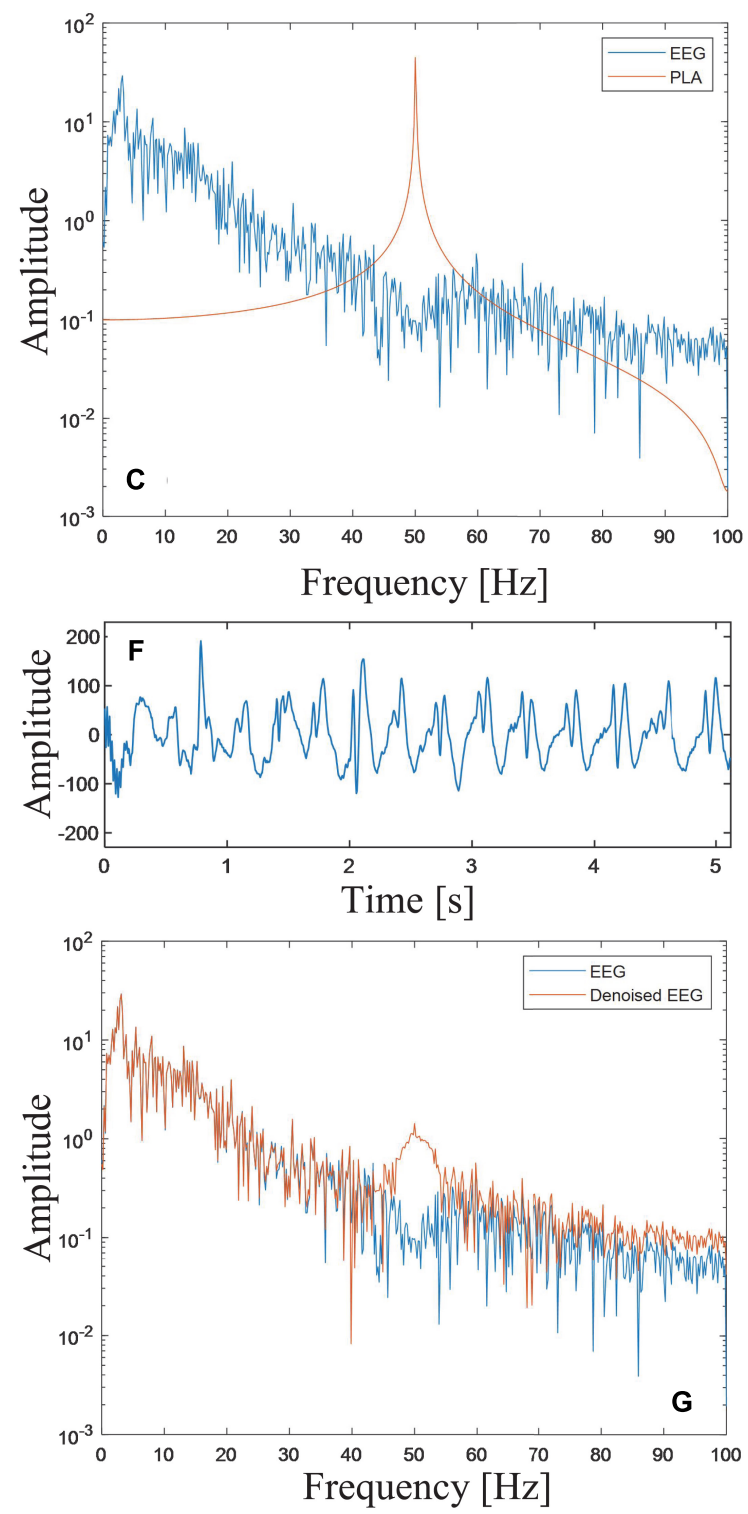

FIGURE 4 | (A) Time domain waveform of the EEG signal; (B) the simulated PLA noise; (C) FFT spectra of the EEG signal and the PLA noise; (D) the denoised signal based on the proposed DSCA; (E) FFT spectra of the EEG signal and the filtered signal using DSCA; (F) the denoised signal based on DNF method; and (G) FFT spectra of the EEG signal and the filtered signal using DNF method.

in Figure 4F. In the time domain waveform, there is a severe distortion on the left side of the filtered signal. The correlation coefficient of the two signals are computed as 0.997. While, from the frequency domain (Figure 4G), differences between the two signals increase significantly near $50 \mathrm{~Hz}$. The above comparisons show that the proposed DSCA algorithm in this paper outperforms DNF in PLA removal.

\section{DISCUSSION}

The problems of energy leakage and PFE are inevitable for harmonic components whose digital samplings do not satisfy the full period sampling condition (Mewett et al., 2001). There is a view that these two side effects can be prevented by actively adjusting the sampling parameters. Because a variation of $\pm 2 \mathrm{~Hz}$ is likely to occur in actual power systems (Singhal et al., 2020), the parameter $f_{\text {pla }}$ is an unknown variable. It is not practical to design DNFs perfectly suitable for all possible PLA noises. On the other hand, increasing the sampling length of EEG signal can improve the accuracy of harmonic parameter estimation. However, the process of brain electrical activity corresponding to the collected signals cannot be repeated, so we can only analyze and process the signals of limited length.

Wavelet transform and EMD outperform FFT by providing time-frequency representation of EEG signal. However, in either 
case, the above side effect is still unavoidable. Because no matter how they decompose the signal, they can only suppress part of the PLA, and the rest will still cause signal distortion. In principle, RBSC uses local spectral information to estimate the overall harmonic composition, so it can avoid the side effect.

The method proposed in this paper is an improvement of the classical RBSC. It not only improves the robustness of the algorithm in parameter correction, but also considers the efficiency of the algorithm. The complexity of the algorithm has only a small increase compared with the classical method. The method proposed in this paper is especially suitable for the case that the value of $\Delta k$ is very small. When the value of this parameter is close to half the frequency resolution, the performance of the two methods is close. In general, the method proposed in this paper can be better applied to the complex measurement environment. However, in any case, the method in this paper has no less accuracy than the original RBSC in statistics. So we recommend that DSCA can completely replace the original RBSC.

Ratio-based spectrum correction only utilize two BSSLs to estimate harmonic parameters, and it can balance the efficiency and accuracy of the algorithm. In the literature, there have been some methods for harmonic parameter estimation using multiple BSSLs. These studies also show that their harmonic parameter estimation accuracy is indeed better than that of RBSC in the absence of noise. But this is not achievable in clinical applications. Therefore, the DSCA is proposed based on the RBSC. The calculation time of RBSC mainly includes the FFT transform of the signal and several complex arithmetic operations. The DSCA proposed in this paper mainly includes two RBSCs, so the efficiency is also very high.

For analysis of a simple harmonic component, the spectral correction is very similar with the spare representation theory

\section{REFERENCES}

An, F. P., Ma, X. M., and Bai, L. (2022). Image fusion algorithm based on unsupervised deep learning-optimized sparse representation. Biomed. Signal Process. Control 71, (Pt B):103140**. doi: 10.1016/j.bspc.2021.10 3140

An, P. F., Yuan, Z. Y., and Zhao, J. H. (2021). Unsupervised multi-subepoch feature learning and hierarchical classification for EEG-based sleep staging. Expert Syst. Appl. 186:18115759. doi: 10.1016/j.eswa.2021.115759

Cao, J. X., Liu, S. J., Liu, H. Q., and Zhang, K. (2021). MRI reconstruction based on Bayesian group sparse representation. Signal Process. 187:108151. doi: 10.1016/ j.sigpro.2021.108151

Cao, X. C., Chen, B. Q., Yao, B., and He, W. P. (2019). Combining translationinvariant wavelet frames and convolutional neural network for intelligent tool wear state identification. Comput. Ind. 106, 71-84. doi: 10.1016/j.compind.2018. 12.018

Chen, B. Q., Lan, Q. X., Li, Y., Zhuang, S. Q., and Cao, X. C. (2019). Enhancement of fault feature extraction from displacement signals by suppressing severe end distortions via sinusoidal wave reduction. Energies 12:3536. doi: 10.3390/ en 12183536

Chen, B. Q., Li, Y., Cao, X. C., Suen, V., and Zhuang, S. (2021). Recovery of undersampled force measurement from high-speed milling process using approximate sparsity in frequency domain. Measurement 175:109143. doi: 10 . 1016/j.measurement.2021.109143

Chen, B. Q., Zhang, Z. S., Sun, C., Li, B., Zi, Y., and He, Z. (2012). Fault feature extraction of gearbox by using overcomplete rational dilation discrete wavelet in mathematical principle. A remarkable advantage is that it does not require a predetermined dictionary. The algorithm can generate a skinny dictionary containing only one atom to represent the actual sinusoidal component. Because the dictionary is not redundant, no iterative algorithms is needed. We can call the spectral correction as implicit sparse representation (ISR) for simple harmonic component analysis. The current research shows that the results obtained by using SR based on iterative numerical algorithm are very similar to those based on spectral correction method. Therefore, in the case of harmonic analysis, a method based on SC may be preferentially employed.

\section{DATA AVAILABILITY STATEMENT}

The data presented in the study are deposited in the ictal repository, accession number ictall (https://www.researchgate.net /publication/308719109_EEG_Epilepsy_Datasets).

\section{AUTHOR CONTRIBUTIONS}

MZ conceived and designed the classification method. Z-FL, RZ, and NX performed the experiment. Z-FL, RZ, Y-QD, and G-HL analyzed the data and wrote the manuscript. MZ and NX reviewed and edited the manuscript. All authors read and approved the manuscript.

\section{FUNDING}

This research is financially supported by the Fundamental Research Funds for the Central Universities (XJS191305).

transform on signals measured from vibration sensors. Mech. Syst. Signal Process. 33, 275-298. doi: 10.1016/j.ymssp.2012.07.007

Collazos-Huertas, D., Caicedo-Acosta, J., Castaño-Duque, G. A., and AcostaMedina, C. D. (2020). Enhanced multiple instance representation using timefrequency atoms in motor imagery classification. Front. Neurosci. 14:155. doi: 10.3389/fnins.2020.00155

Cury, C., Maurel, P., Gribonval, R., and Barillot, C. (2020). A sparse EEG-Informed fMRI model for Hybrid EEG-fMRI neurofeedback prediction. Front. Neurosci. 13:1451. doi: 10.3389/fnins.2019.01451

Dagdevir, E., and Tokmakci, M. (2021). Optimization of preprocessing stage in EEG based BCI systems in terms of accuracy and timing cost. Biomed. Signal Process. Control 67:102548. doi: 10.1016/j.bspc.2021.102548

Deshpande, G., Rangaprakash, D., Oeding, L., Cichocki, A., and Hu, X. P. (2017). A new generation of brain-computer interfaces driven by discovery of latent EEG-fMRI linkages using tensor decomposition. Front. Neurosci. 11:246. doi: 10.3389/fnins.2017.00246

Faiman, I., Smith, S., Hodsoll, J., Young, A. H., and Shotbolt, P. (2021). Restingstate EEG for the diagnosis of idiopathic epilepsy and psychogenic nonepileptic seizures: a systematic review. Epilepsy Behavior. 121:108047. doi: 10.1016/j. yebeh.2021.108047

He, W. P., Zi, Y. Y., Chen, B. Q., Wu, F., and He, Z. J. (2015). Automatic fault feature extraction of mechanical anomaly on induction motor bearing using ensemble super-wavelet transform. Mech. Syst. Signal Process. 54-55, 457-480. doi: 10.1016/j.ymssp.2014.09.007

Hernández-Ronquillo, L., Thorpe, L., Dash, D., Hussein, T., Hunter, G., Waterhouse, K., et al. (2020). Diagnostic accuracy of the ambulatory EEG vs. 
routine EEG for first single unprovoked seizures and seizure recurrence: the DX-seizure study. Front. Neurol 11:223. doi: 10.3389/fneur.2020.00223

Hong, K.-S., Khan, M. J., and Hong, M. J. (2018). Feature extraction and classification methods for Hybrid fNIRS-EEG brain-computer interfaces. Front. Hum. Neurosci. 12:246. doi: 10.3389/fnhum.2018.00246

Huang, J. S., Chen, B. Q., Li, Y., and Sun, W. F. (2021). Fractal geometry of wavelet decomposition in mechanical signature analysis. Measurement 173:108571. doi: 10.1016/j.measurement.2020.108571

Huang, J. S., Chen, B. Q., Yao, B., and He, W. (2019). ECG arrhythmia classification using STFT-based spectrogram and convolutional neural network. IEEE Access 7, 92871-92880. doi: 10.1109/ACCESS.2019.2928017

Jana, R., and Mukherjee, I. (2021). Deep learning based efficient epileptic seizure prediction with EEG channel optimization. Biomed. Signal Process. Control 68:102767. doi: 10.1016/j.bspc.2021.102767

Leske, S., and Dalal, S. S. (2019). Reducing power line noise in EEG and MEG data via spectrum interpolation. NeuroImage 189, 763-776. doi: 10.1016/j. neuroimage.2019.01.026

Li, M. Y., and Chen, W. Z. (2021). FFT-based deep feature learning method for EEG classification. Biomed. Signal Process. Control 66:102492. doi: 10.1016/j. bspc.2021.102492

Li, Y. F., and Chen, K. F. (2008). Eliminating the picket fence effect of the fast Fourier transform. Comput. Phys. Commun. 178, 486-491. doi: 10.1016/j.cpc. 2007.11.005

Mallat, S. G. (1989). A theory for multiresolution signal decomposition : the wavelet representation. IEEE Trans. Pattern Anal. Mach. Intell. 11, 674-693. doi: $10.1109 / 34.192463$

Mewett, D. T., Nazeran, H., and Reynolds, K. J. (2001). "Removing power line noise from recorded EMG," in Proceedings of the 23rd Annual International Conference of the IEEE, Istanbul, doi: 10.1109/IEMBS.2001.1017205

Miao, Y., Jurica, P., Struzik, Z. R., Hitomi, T., Kinoshita, A., Takahara, Y., et al. (2021). Dynamic theta/beta ratio of clinical EEG in Alzheimer's disease. J. Neurosc. Methods 359:109219. doi: 10.1016/j.jneumeth.2021.109219

Piskorowski, J. (2013). Time-efficient removal of power-line noise from EMG signals using IIR notch filters with non-zero initial conditions. Biocybern. Biomed. Eng. 33, 171-178. doi: 10.1016/j.bbe.2013.07.006

Prathaban, B. P., and Balasubramanian, R. (2021). Dynamic learning framework for epileptic seizure prediction using sparsity based EEG reconstruction with optimized CNN classifier. Expert Syst. Appl. 170:114533. doi: 10.1016/j.eswa. 2020.114533

Saegh, A. A., Dawwd, S. A., and Jabbar, J. M. A. (2021). Deep learning for motor imagery EEG-based classification: a review. Biomed. Signal Process. Control 63:102172. doi: $10.1016 /$ j.bspc.2020.102172

Shankar, A., Khaing, H. K., Danpapat, S., and Barma, S. (2021). Analysis of epileptic seizures based on EEG using recurrence plot images and deep learning. Biomed. Signal Process. Control 69:102854. doi: 10.1016/j.bspc.2021.102854

Sharma, G., Paashar, A., and Joshi, A. M. (2021). DepHNN: a novel hybrid neural network for electroencephalogram (EEG)-based screening of depression. Biomed. Signal Process. Control 66:102393. doi: 10.1016/j.bspc.2020.102393

Sharma, S., Khare, S. K., Bajaj, V., and Ansari, I. A. (2021). Improving the separability of drowsiness and alert EEG signals using analytic form of wavelet transform. Appl. Acoust. 181:108164. doi: 10.1016/j.apacoust.2021.10 8164

Singhal, A., Singh, P., Fatimah, B., and Pachori, R. B. (2020). An efficient removal of power-line interference and baseline wander from ECG signals by employing Fourier decomposition technique. Biomed. Signal Process. Control 57:101741. doi: 10.1016/j.bspc.2019.101741

Sun, Y., Wei, C., Cui, V., Xiu, M., and Wu, A. (2020). Electroencephalography: clinical applications during the perioperative period. Front. Med. 7:251. doi: 10.3389/fmed.2020.00251

Taran, S., Bajaj, V., Sharma, D., Siuly, S., and Segur, A. (2018). Features based on analytic IMF for classifying motor imagery EEG signals in BCI applications. Measurement 116, 68-76. doi: 10.1016/j.measurement.2017.10.067

Wang, C., Kipping, J., Bao, C., Ji, H., and Qiu, A. (2016). Cerebellar functional parcellation using sparse dictionary learning clustering. Front. Neurosci. 10:188. doi: 10.3389/fnins.2016.00188

Wu, Z. H., and Huang, N. E. (2004). A study of the characteristics of white noise using the empirical mode decomposition method. Proc. R. Soc. Lond. Ser. Math. Phys. Eng. Sci. 460, 1597-1611. doi: 10.1098/rspa.2003.1221

Yang, G. A., Yang, J. J., Lu, Z. Z., and Liu, D. Y. (2020). A convolutional neural network with sparse representation. Knowl. Based Syst. 209:106419. doi: 10. 1016/j.knosys.2020.106419

Yang, Z., Zhuang, X., Bird, C., Sreenivasan, K., Mishra, V., Banks, S., et al. (2019). Performing sparse regularization and dimension reduction simultaneously in multimodal data fusion. Front. Neurosci. 13:642. doi: 10.3389/fnins.2019. 00642

Zhang, Z. Q., Yang, Q. Y., and Zi, Y. Y. (2021). Multi-scale and multi-pooling sparse filtering: a simple and effective representation learning method for intelligent fault diagnosis. Neurocomputing 451, 138-151. doi: 10.1016/j.neucom.2021.04. 066

Conflict of Interest: J-LT, Z-FL, and RZ were employed by the company Shaanxi Aerospace Technology Application Research Institute Co., Ltd.

The remaining authors declare that the research was conducted in the absence of any commercial or financial relationships that could be construed as a potential conflict of interest.

Publisher's Note: All claims expressed in this article are solely those of the authors and do not necessarily represent those of their affiliated organizations, or those of the publisher, the editors and the reviewers. Any product that may be evaluated in this article, or claim that may be made by its manufacturer, is not guaranteed or endorsed by the publisher.

Copyright (c) 2021 Tan, Liang, Zhang, Dong, Li, Zhang, Wang and Xu. This is an open-access article distributed under the terms of the Creative Commons Attribution License (CC BY). The use, distribution or reproduction in other forums is permitted, provided the original author(s) and the copyright owner(s) are credited and that the original publication in this journal is cited, in accordance with accepted academic practice. No use, distribution or reproduction is permitted which does not comply with these terms. 